\title{
Congenital Adrenal Hyperplasia: A Possible Risk Factor for Neonatal Adrenal Hemorrhage
}

\author{
Sota Iwatani ${ }^{\mathrm{a}, \mathrm{b}}$, Emiko Takeoka ${ }^{\mathrm{a}}$, Masami Mizobuchi ${ }^{\mathrm{a}}$, Seiji Yoshimoto ${ }^{\mathrm{a}}$, Hideto Nakao ${ }^{\mathrm{a}}$
}

\begin{abstract}
Here, we describe the first case of neonatal adrenal hemorrhage (AH) complicated with congenital adrenal hyperplasia (CAH). Our findings suggest that adrenal glands that have enlarged due to $\mathrm{CAH}$ are more susceptible to hemorrhaging during labor, and therefore, that $\mathrm{CAH}$ could be a risk factor for $\mathrm{AH}$.
\end{abstract}

Keywords: Congenital adrenal hyperplasia; Adrenal hemorrhage; Ultrasound; Adrenal gland

\section{Introduction}

The etiology of adrenal hemorrhage (AH) is unknown, but the adrenal cortex at birth is relatively large, extremely vascular, and prone to trauma; venous engorgement has been proposed as a mechanism. Here, we demonstrate the first case of neonatal AH complicated with congenital adrenal hyperplasia $(\mathrm{CAH})$.

\section{Case Report}

A 3,150 g newborn male was delivered after an uncompli-

Manuscript accepted for publication August 7, 2014

${ }^{\text {a}}$ Department of Neonatology, Hyogo Prefectural Kobe Children's Hospital Perinatal Center, 1-1-1 Takakuradai, Suma-Ku, Kobe-Shi, Hyogo 654-0081, Japan

${ }^{\mathrm{b}}$ Corresponding Author: Sota Iwatani, Department of

Neonatology, Hyogo Prefectural Kobe Children's Hospital Perinatal Center, 1-1-1 Takakuradai, Suma-Ku, Kobe, Hyogo 654-0081, Japan.

Email: iwatani_kch@hp.pref.hyogo.jp

doi: http://dx.doi.org/10.14740/jmc1909w cated pregnancy via vaginal delivery as the second child of unrelated healthy parents. His brother was healthy. At $3 \mathrm{~h}$ after birth, he was admitted for tachypnea and dusky skin discoloration. A physical examination revealed a right-sided abdominal mass. Abdominal ultrasound detected a large loculated collection of fluid in the right suprarenal region (Fig. 1A). The patient's jaundice progressed rapidly despite intensive phototherapy, and a second ultrasound scan demonstrated that the fluid collection had reduced in size and exhibited mixed echogenicity (Fig. 1B). Abdominal computed tomography demonstrated a low-density lesion in the right suprarenal region (Fig. 2). These findings were consistent with a right $\mathrm{AH}$. The left adrenal gland was enlarged $(24.0 \times$ $6.0 \mathrm{~mm}$ in size; normal mean size: $14.4 \times 1.9 \mathrm{~mm}$ [1]) (Fig. 1C). Although the patient's jaundice improved, his condition slowly deteriorated, and marked weight loss was observed. On the 10th day, a laboratory examination revealed hyponatremia (Na: $133 \mathrm{mEq} / \mathrm{L})$ and hyperkalemia $(\mathrm{K}: 6.6 \mathrm{mEq} / \mathrm{L})$. Furthermore, the patient's serum 17-hydroxyprogesterone level was elevated $(590 \mathrm{ng} / \mathrm{dL})$. These results were indicative of the classical type of CAH due to 21-hydroxylase deficiency. Hydrocortisone treatment was initiated, and the patient's condition improved markedly. He was discharged after being prescribed hydrocortisone and 9-alpha-fludrocortisone. Subsequently, mutations in the steroid 21-hydroxylase gene were detected.

\section{Discussion}

In neonates, the adrenal gland is vulnerable to hemorrhaging because of its large size and high vascularity [1]. AH is an uncommon condition in the neonatal period (incidence: $0.2 \%$ ). It can be caused by a difficult labor [2]; however, in some cases its etiology is unknown [3]. The clinical features of $\mathrm{AH}$ are variable and non-specific [2]. $\mathrm{AH}$ rarely leads to transient adrenal insufficiency [2,3]; however, no cases of AH complicated with CAH have been reported in the medical literature. We suggest that adrenal glands that have enlarged due to $\mathrm{CAH}$ are more susceptible to hemorrhaging during labor, and therefore, that $\mathrm{CAH}$ could be a risk factor for $\mathrm{AH}$. 


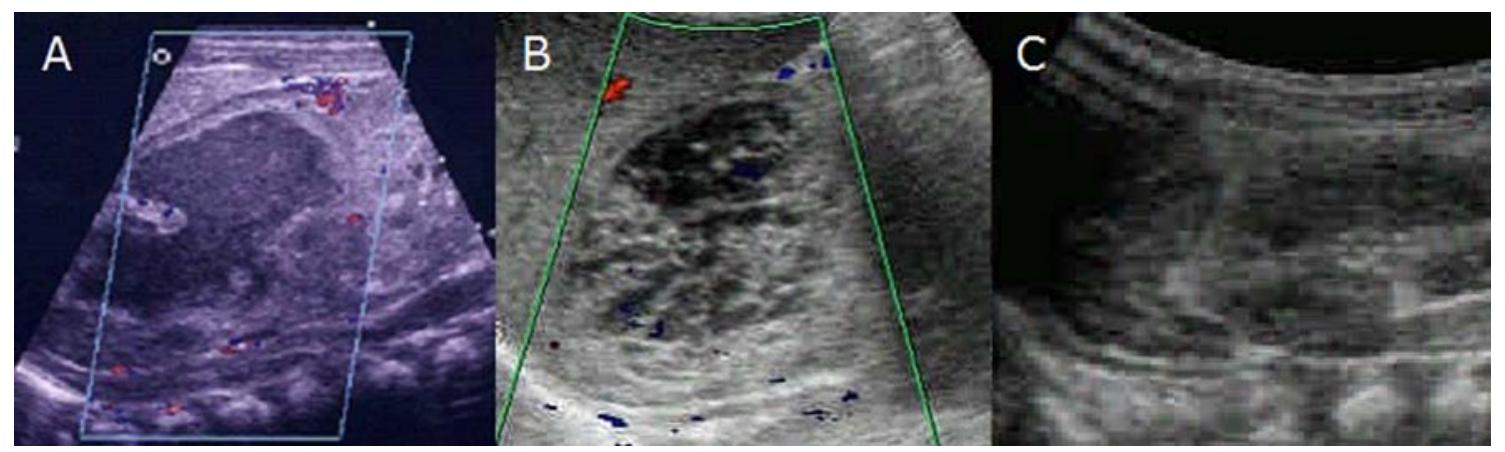

Figure 1. (A) Para-sagittal section through the partly liquefied right-sided adrenal hemorrhage on the day of admission. An examination of vascular flow did not detect any blood flow foci within the hemorrhagic region of the adrenal gland. (B) A follow-up examination performed at 3 days after birth demonstrated a reduction in the size of the right AH. (C) During the examination mentioned in $B$, it was also found that the left adrenal gland was enlarged $(24.0 \times 6.0 \mathrm{~mm})$.

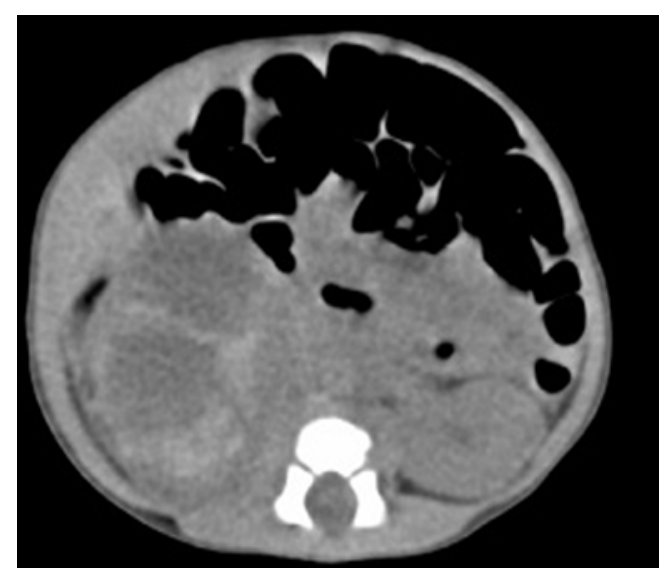

Figure 2. On the third day, abdominal computed tomography detected a lobulated low-density lesion in the right suprarenal region.

\section{Acknowledgement}

We thank the patient's family for their kind cooperation and for giving us permission to publish this case report.

\section{Author Contributions}

Sota Iwatani M.D. and Emiko Takeoka M.D. contributed equally to this work.

\section{Conflict of Interest}

The authors have no conflicts of interest to declare.

\section{References}

1. Westra SJ, Zaninovic AC, Hall TR, Kangarloo H, Boechat MI. Imaging of the adrenal gland in children. Radiographics. 1994;14(6):1323-1340.

2. Mutlu M, Karaguzel G, Aslan Y, Cansu A, Okten A. Adrenal hemorrhage in newborns: a retrospective study. World J Pediatr. 2011;7(4):355-357.

3. Koklu E, Kurtoglu S, Akcakus M, Koklu S. Adrenal haemorrhage with cholestasis and adrenal crisis in a newborn of a diabetic mother. J Pediatr Endocrinol Metab. 2007;20(3):441-444. 